\title{
Application and Development of 3D Printing in Medical Field
}

\author{
Chunhua Sun, Guangqing Shang \\ Department of Mechanical and Electrical Engineering, Suzhou Vocational University, Suzhou, China \\ Email:chh_sunny@163.com
}

How to cite this paper: Sun, C.H. and Shang, G.Q. (2020) Application and Development of 3D Printing in Medical Field. Modern Mechanical Engineering, 10, 25-33. https://doi.org/10.4236/mme.2020.103003

Received: July 2, 2020

Accepted: August 7, 2020

Published: August 10, 2020

Copyright $\odot 2020$ by author(s) and Scientific Research Publishing Inc. This work is licensed under the Creative Commons Attribution International License (CC BY 4.0).

http://creativecommons.org/licenses/by/4.0/

\begin{abstract}
Different from reduction manufacturing and equal manufacturing, 3D printing is an additive manufacturing method, which transforms 3D model into $2 \mathrm{D}$ cross-section data to form entity layer by layer. This makes its processing not limited by complexity of the design model and number of the manufacturing products. It is very suitable for the medical field with high customization requirements. In fact, application of 3D printing technology in the medical field is particularly noticeable. In this paper, application and development of 3D printing technology are reviewed in medical model, rehabilitation equipment, tissue engineering, medical hygiene materials, lab-on-chip. Its applications include medical education, surgical planning, prosthesis customization, tissue culture and biosensor manufacturing and so on. Its wide application is due to its digital model, which makes the whole manufacturing process easier to digitize, so it is more conductive to updating and customization of products via $3 \mathrm{D}$ printing.
\end{abstract}

\section{Keywords}

3D Printing, Additive Manufacturing, Medical Field

\section{Introduction}

3D printing technology uses additive manufacturing to complete forming of $3 \mathrm{D}$ entity via layer by layer, which makes the whole process not limited by the complexity of design structure. Therefore, 3D printing has been applied in many fields. As one of representative technologies of the fourth industrial revolution, 3D printing is profoundly affecting all aspects and embodies extraordinary value. The main application value of 3D printing technology in the medical field is to provide better personalized treatment for patients with efficient and accurate digital design and manufacturing means owing to additive manufacturing mode. 
At present, 3D printing in orthopedic implants and dentistry is stepping into industrialization, and it is also widely used in medical devices, medical education, operation planning, bioactive sensor and so on. The general development trend of $3 \mathrm{D}$ printing in the medical field is from non-bioactive medical devices to bioactive artificial organs [1]. In this paper, application and development of 3D printing technology are reviewed from some aspects of medical model, rehabilitation equipment, tissue engineering, medical hygiene materials, lab-on-chip. Further, a summary is given to point out some problems and development trend of $3 \mathrm{D}$ printing in medical field.

\section{Application and Development of 3D Printing Technology in Medical Field}

\subsection{D Printing Medical Model}

One of the most popular uses of 3D printing is in dentistry. As there are many reports on this aspect, this paper will not review it. In addition, another wide application of $3 \mathrm{D}$ printing is in medical education and operation planning. Based on the image data collected by CT, MRI and other scanning equipment, the 3D printing medical model can be used for operation pre-planning, medical education and doctor-patient communication. Figure 1 shows models of new coronavirus and the infected lung, which is used to popularize the knowledge of new coronavirus. More and more medicine subjects, such as orthopedics, neurosurgery and oncology, use 3D printing medical model to pre-plan operation and improve success rate of the operation and reduce the risk. The more difficult the operation meets, the more valuable to plan the operation through $3 \mathrm{D}$ printing model is [2]. A typical application is in the accurate resection of mediastinal tumor. In order to avoid damaging the surrounding important tissues such as trachea, cardiovascular and nerve when cutting the deep tumor, the double source spiral CT was used to obtain data and make the 3D printing model of the tumor and its adjacent organs. The best operation path and approaching skills were explored in the pre-operation to improve the success rate of operation [3].

At present, 3D printing technology in medical model needs to be improved in the following two aspects: one is $3 \mathrm{D}$ medical modeling technology, the other is

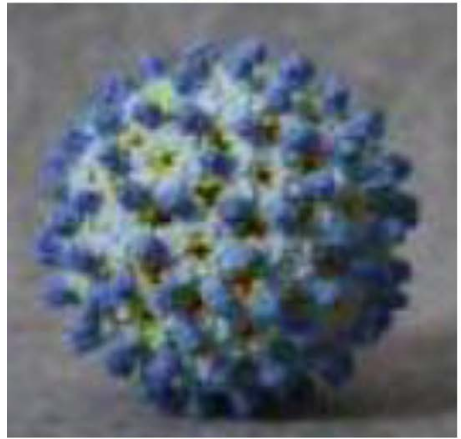

(a)

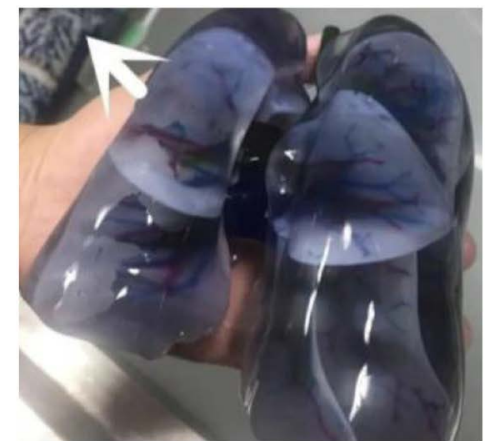

(b)

Figure 1. Medical model via 3D printing technology. (a) Coronavirus; (b) Infected lung. 
the integration among medical modeling, 3D printing process and medical diagnosis process [4].In order to solve the existing medical modeling problems of time-consuming, tedious and low resolution, scientists proposed a halftone method [5].This method could keep high level anatomical details completely, make complex medical images easier and faster to be read by $3 \mathrm{D}$ printing equipment. At the same time, this method could support 3D printer to manufacture with two different printing materials.

Up to now, the new mode of seamless integration of data collection, model reconstruction and $3 \mathrm{D}$ printing has gradually replaced the original isolated mode. Philips has adopted the new mode in its current medical imaging and software developing. GE is studying how to combine its advanced CT equipment with 3D printing devices to produce medical model quickly. At the same time, GE has also established strategic cooperation with Stratasys Company to lay a solid foundation for seamless integration between medical model data collection and 3D printing [6].

\subsection{Implants}

Implants are also a major aspect of 3D printing technology. In clinical treatment, implant is one of the treatment methods of skeletal muscle system. It can replace joint, bone, cartilage or musculoskeletal system in whole or part to avoid the mismatch of prosthesis size. Figure 2 illustrates some metal medical applications

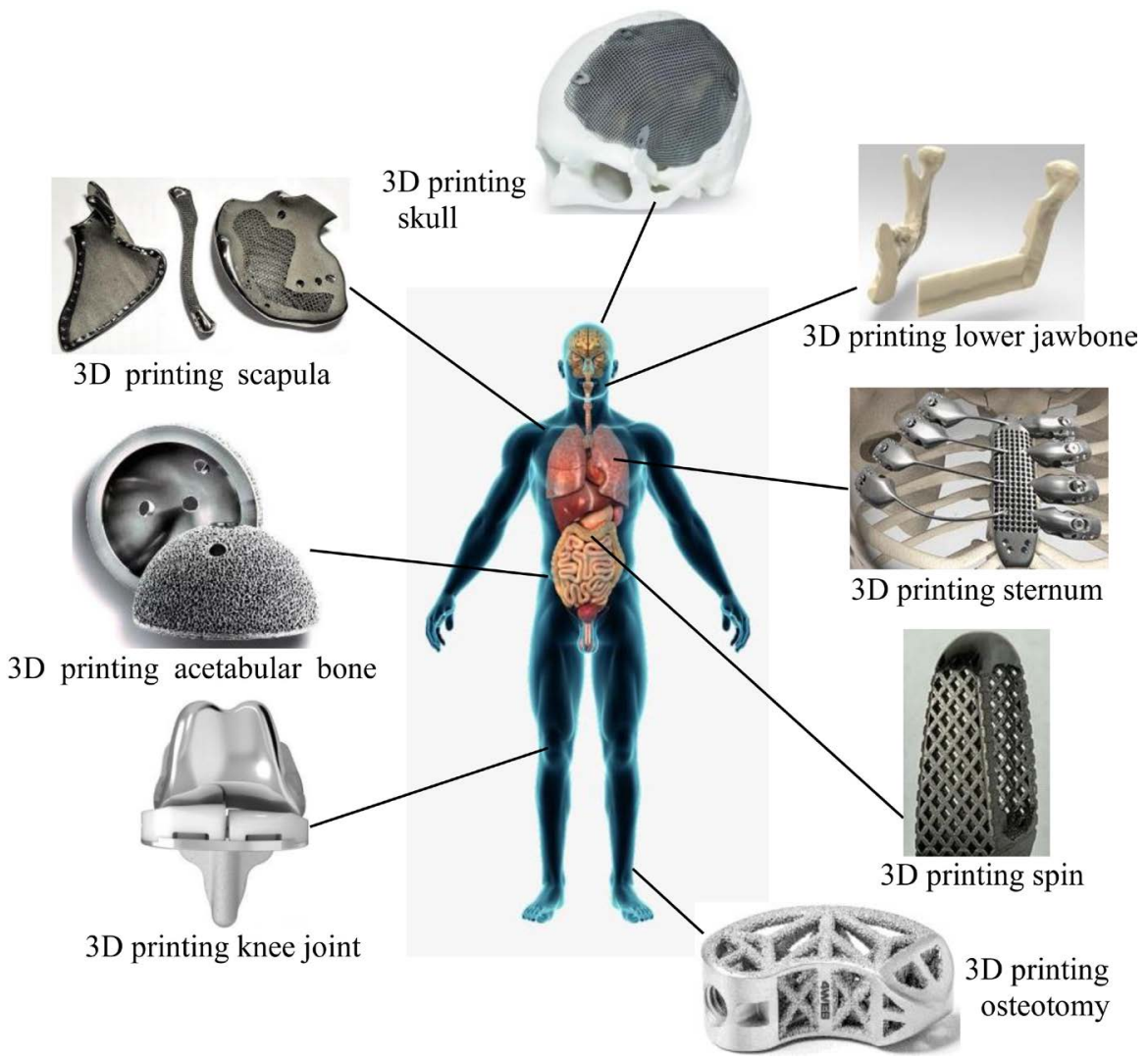

Figure 2. Some metal implants via 3D printing. 
of 3D printing implants. Using 3D printing to make implants has the advantages of short cycle, low cost, customization, accurate completion of bionic porous structure, absorption of human bone cells, promotion of bone integration of implants, and avoidance of risk of surface coating falling off. At present, some 3D printing implants have been marketed, including knee joint, posterior lumbar interbody fusion device, meniscus tissue, spine, hip joint, knee joint bracket, etc. There are three kinds of materials used to 3D printing implants: metal, ceramic and polymer. The latter two materials can be further subdivided into degradable and non-degradable materials. Degradable implants will be gradually decomposed into components that can be absorbed by human body in a certain period after being implanted into human body. The application of 3D printing technology can be directly or indirectly used to manufacture implants. The indirect manufacturing mode is that $3 \mathrm{D}$ print casting mold is firstly done and the implants by traditional casting process are finally produced. The mode is more suitable for the mass production of the same type of products.

Wong et al. designed and printed the implant according to the shape and biomechanical analysis of pelvic defect after tumor resection [7]. The operation result showed that the patients could walk independently and the hip joint recovered well. In order to promote the fusion and reduce the stiffness mismatch between bone cells and implants, Shah et al. simulated the ultra-structure of natural bone canaliculus, designed a porous channel [8]. The morphology of bone cells showed that the $3 \mathrm{D}$ printed implants grew well with the human interface tissue, which was helpful to eliminate the stress shielding effect and promote the bone healing better. In addition, 3D printing elastic film implants can be used to treat heart disease, and 3D printing nerve guide tubes can be used to guide the nervous system to finish self-repairing [9].

\subsection{Rehabilitation Equipment}

Application of 3D printing technology of rehabilitation equipment is mainly taken in orthopedics, prosthetics, and hearing aids and so on. In the manufacturing orthosis, there are four kinds of 3D printing technology, including selective laser sintering (SLS), material spraying, melting deposition molding and photo polymerization. No matter which one is chosen, it has the characteristics of lightweight and customization. It is the most common 3D printing technology for orthosis to adopt SLS via nylon material. As shown in Figure 3, a 3D printing foot orthosis with integrated functions was manufactured by SLS. It has various geometric structures, and the annular sealing system covers almost the whole foot surface to prevent excessive sweating. The 3D printed lower limb orthosis was made of rigid materials with multi-material spraying process via $3 \mathrm{D}$ printing [10]. It adopted an integrated structure with many hollows in the main body. Therefore, it possesses characteristics of light weight and good ventilation. 3D printing orthotics with the materials of $\mathrm{ABS}$, peek and other engineering plastics are manufactured through melting deposition, as shown in Figure 4 [11]. It can provide better 
support and stability in the rehabilitation process and further reduce the knee impact by the biomechanical design.

The application of 3D printing technology in the upper limb prosthesis includes the personalized shell of biomimetic EMG hand and some low-cost manipulator. Meanwhile, application of the lower limb prosthesis is mainly in the customized shell and foot device. In the lower leg prosthesis, receiving cavity and shell by integrated 3D printing prosthesis designing has also appeared.

Since the beginning of the $21^{\text {st }}$ century, 3D printing technology has been used in mass customization production of hearing aid shell which DLP photo-polymerization process is taken and the printing material is photosensitive resin. Selective laser melting technology and titanium alloy material are further used to reduce the volume of hearing aid and improve the strength of its shell.

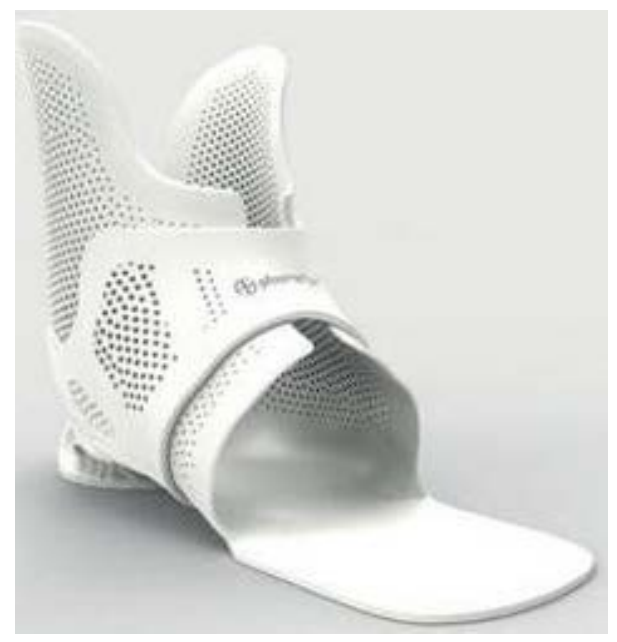

Figure 3. Integrated function orthosis via $3 \mathrm{D}$ printing.
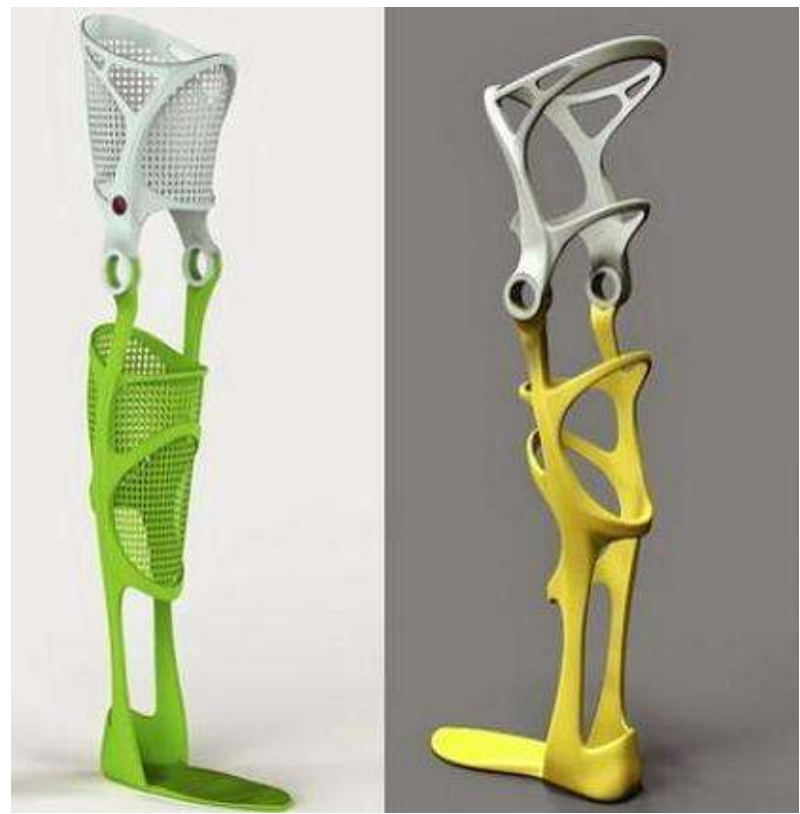

Figure 4. Leg orthosis via 3D printing. 


\subsection{Surgical Assistant Instruments}

$3 \mathrm{D}$ printing navigation template is a kind of surgical tool which is based on the anatomical structure of patients [12]. In minimally invasive percutaneous nephrolithotomy, osteotomy and puncture operation, 3D printing navigation template has played an important role in improving accuracy of operation, reducing risk of operation and shortening operation time [13] [14].

\subsection{Tissue Engineering}

3D printing scaffolds have excellent properties in high porosity, sufficient pore size, appropriate mechanical properties, biodegradability and biocompatibility. This kind of scaffolds have been applied in many fields such as nerve repair, vascular repair, cartilage tissue repair, heart valve repair [15] [16]. However, there are few studies on biocompatibility of the scaffolds. In the latest study, a polycaprolactone PTS scaffold was prepared by $3 \mathrm{D}$ printing technology and surface modification of nano-silica. HE staining showed that the cell affinity of the modified PTS scaffolds was significantly increased, and the immune rejection of animals after acute phase was significantly decreased, which indicated that the tissue compatibility of the modified PTS scaffolds was very well [17].

Tissue regeneration and organ transplantation using 3D bio-printing are hot topics in medical field. At present, 3D printed artificial cornea similar to natural corneal stroma with appropriate scaffold has been made by using the existing 3D digital human corneal model, which has been applied in the field of corneal tissue engineering [18]. The mixed 3D cell printing system with ink-jet coating module to evenly distribute the cuticle, which was supported by polycaprolactone mesh structure, was used to manufacture skin model. And the dermis was filled with layer fiber cells on the skin model. The formed skin model showed good biological characteristics [19]. The 3D bio-printing technology has been used to form liver, heart, cartilage and vascular system. But the feasibility of this technology needs further to be studied [20].

\subsection{Medical Hygiene Materials}

Using 3D printing technology, personalized wound dressing are made by integrating antibacterial silver, copper and zinc into polycaprolactone wire. The application shows good germicidal effect [21]. Using the same method, the 3D printing polyoxymethylene siloxane bionic nano-silver wound dressing also shows good biocompatibility, flexibility and anti-adhesion ability, which can effectively antibacterial and promote wound healing [22].

\subsection{Lab-on-Chip}

Lab-on-chip is a new technology that integrates the biological and chemical laboratory on a chip of micrometer scale to carry out detection and analysis. As early as 2010,3D printing polyjet technology was firstly used for indirect manufacturing microfluidic chips. The application of directly one-time molding and manufacturing microfluidic chips by $3 \mathrm{D}$ printing technology has gradually been 
emerging from 2011. Figure 5 shows the commonly 3D printing technology of microfluidic chip. The microfluidic chip made by 3D printing technology was made to detect hepatitis B virus nucleic acid by fluorescence quantitative polymerase chain reaction, which detection performance surpasses the commonly used nucleic acid blood screening system [23]. The 3D printing biosensor can be used to detect metallothionein as a tumor marker by fluorescence, which opens up a new way for tumor diagnosis [24].

At present, 3D printing technology in lab-on-chip is still in the early stage of application and exploration. Its mainly applications are chip development and design verification. In the future, 3D printing technology will play a greater role in the integration and miniaturization as well as the production of lab-on-chip for real-time diagnosis.

\section{Summary}

To sum up, 3D printing technology has developed rapidly and has been many applications in medical fields due to its digital model, which makes the whole manufacturing process easier to digitize and automate. 3D printing technology has made new breakthroughs in the fields of 3D biological printing and virtual medical treatment. Although these researches have not been applied to human body, some of them have been successful in animal experiments. The feasibility of $3 \mathrm{D}$ printing technology needs a lot of experimental and clinical data to prove.

With the emergence of new medical printing materials and new printing processes, 3D printing technology can not only be used to produce physiological organs and tissues for repairing and regenerating damaged tissues, but also manufacturing protein carriers at the micro level. Through the protein carriers, it provides a new idea to solve the problems such as metabolic disorders of human biochemical substances.

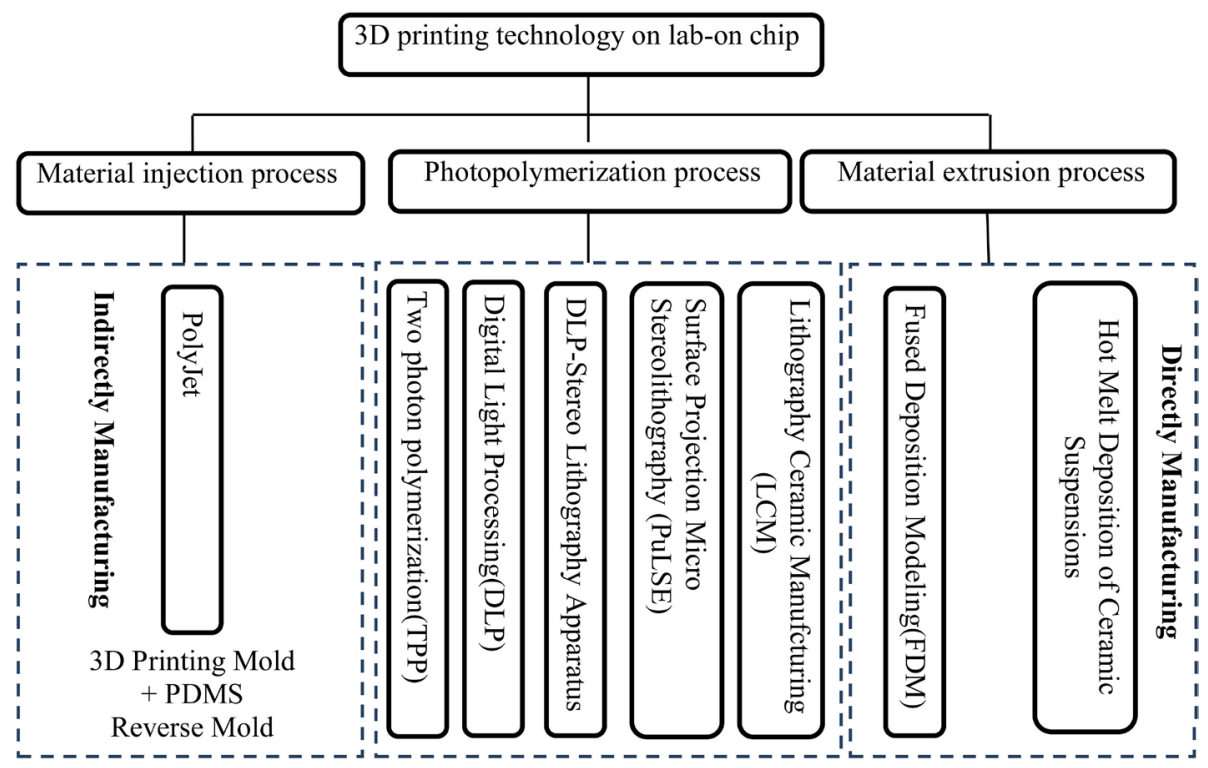

Figure 5. Lab-on-chip via 3D printing technology. 


\section{Acknowledgements}

This work was supported by "3C-Product Intelligent Manufacturing Engineering Technology Research and Development Center of Jiangsu Province" from Jiangsu Education Department and "New Driving and Ambient Energy Harvesting Innovation Team Based on Functional Materials" from Suzhou Vocational University.

\section{Conflicts of Interest}

The authors declare no conflicts of interest regarding the publication of this paper.

\section{References}

[1] Wu, H.Y. (2017) 3D Printing: Three-Dimensional Creation via Intelligent Digitization. Publishing House of Electronic Industry, Beijing.

[2] Wang, D.Q. and Li, B. (2016) Development of Application Study on 3D Printing Technology in Medical Field. Chongqing Medicine, 45, 126-128.

[3] Sun, T., Han, S.Q., Wang, J.W., et al. (2018) Value of 3D Printing Technology in Precise Resection of Mediastinal Tumors. Chinese Medical Equipment Journal, 39, 54-56, 78.

[4] Hu, J.Y., Li, Y.W., Zhang, Y.Q., et al. (2019) Application Progress of 3D Printing Technology in Clinical Medicine. Shandong Medical Journal, 59, 106-109.

[5] Harvard University and Massachusetts Institute of Technology Jointly Use Halftone Method to Greatly Shorten the Process from Scanning to 3D Printing. https://www.sohu.com/a/238191648 99940258

[6] Song, Y. and Wang, K. (2019) Analysis on the Innovation Ecosystem and Characteristics of 3D Printing Enterprise-Case Study on 3D Systems and Stratasys. Journal of UESTC (Social Sciences Edition), 2, 85-93.

[7] Wong, K.C., Kumta, S.M., Geel, N.V., et al. (2015) One-Step Reconstruction with a 3D-Printed, Biomechanically Evaluated Custom Implant after Complex Pelvic Tumor Resection. Computer Aided Surgery, 20, 14-23. https://doi.org/10.3109/10929088.2015.1076039

[8] Shah, F.A., Snis, A., Matic, A., et al. (2016) 3D Printed Ti6Al4V Implant Surface Promotes Bone Maturation and Retains a Higher Density of Less Aged Osteocytes at the Bone-Implant Interface. Acta Biomaterialia, 30, 357-367. https://doi.org/10.1016/j.actbio.2015.11.013

[9] Gao, X.B., Wang, L.H. and Liu, H.H. (2017) Main Application of 3D Printing Technology in Medical Industry. Smart Health, 3, 5-8.

[10] Banks, J. (2013) Adding Value in Additive Manufacturing Researchers in the United Kingdom and Europe Look to 3D Printing for Customization. IEEE Pulse, 4, 22-26. https://doi.org/10.1109/MPUL.2013.2279617

[11] Xiong, B.L., Zhou, D.W., et al. (2018) Application Prospect of 3D Printing in Prosthetic Orthosis Technology. Rehabilitation in China, 33, 523-525.

[12] Wang, R., Lu, L.Z. and Lin, J. (2017) Application Progress of 3D Printing Technology in Precise and Minimally Invasive Treatment. Chinese Journal of Pain Medicine, 23, 81-85. 
[13] Golab, A., Smektala, T., Krolikowski, M., et al. (2018) Percutaneous Nephrolithotomy Using an Individual 3-Dimensionally Printed Surgical Guide. Urologia Internationalis, 100, 485-487. https://doi.org/10.1159/000446291

[14] Mcallister, P., Watson, M. and Burke, E. (2018) A Cost-Effective, In-House, Positioning and Cutting Guide System for Orthognathic Surgery. Journal of Maxillofacial and Oral Surgery, 17, 112-114. https://doi.org/10.1007/s12663-017-1067-y

[15] Lee, J.W. and Cho, D.W. (2015) 3D Printing Technology over a Drug Delivery for Tissue Engineering. Current Pharmaceutical Design, 21, 1606-1617. https://doi.org/10.2174/1381612821666150115125324

[16] Zhu, M., Huang, T., et al. (2017) Progress in 3D Printing of Biomaterials. Shanghai University of Science and Technology, 39, 473-483, 489.

[17] Pan, S., Zhong, Y., Shan, Y., et al. (2019) Selection of the Optimum 3D-Printed Pore and the Surface Modification Techniques for Tissue Engineering Tracheal Scaffold in Vivo Reconstruction. Journal of Biomedical Materials Research Part A, 107, 360-370. https://doi.org/10.1002/jbm.a.36536

[18] Isaacson, A., Swioklo, S. and Connon, C.J. (2018) 3D Bioprinting of a Corneal Stroma Equivalent. Experimental Eye Research, 173, 188-193.

https://doi.org/10.1016/j.exer.2018.05.010

[19] Kim, B.S., Lee, J.S., Gao, G., et al. (2017) Direct 3D Cell-Printing of Human Skin with Functional Transwell System. Biofabrication, 9, Article ID: 025034. https://doi.org/10.1088/1758-5090/aa71c8

[20] Zhang, Y.S., Yue, K., Aleman, J., et al. (2017) 3D Bioprinting for Tissue and Organ Fabrication. Annals of Biomedical Engineering, 45, 148-163. https://doi.org/10.1007/s10439-016-1612-8

[21] Muwaffak, Z., Goyanes, A., Clark, V., et al. (2017) Patient-Specific 3D Scanned and 3D Printed Antimicrobial Polycaprolactone Wound Dressings. International Journal of Pharmaceutics, 527, 161-170. https://doi.org/10.1016/j.ijpharm.2017.04.077

[22] Shi, G.Q., Luo, G.X., Chen, X.W., et al. (2018) 3D Printing Polyoxymethylene Siloxane Biomimetic Silver Nanocomposites and Their Properties. Medical Journal of Chinese People's Liberation Army, 43, 840-848.

[23] Wang, K.K., Yang, K., Zhao, J., et al. (2018) Rapid Detection of Hepatitis B Virus Nucleic Acid by Fluorescence Quantitative PCR Based on Microfluidic Chip. Journal of Analytical Science, 34, 450-454.

[24] Heger, Z., Zitka, J., Cernei, N., et al. (2015) 3D-Printed Biosensor with Poly (dimethylsiloxane) Reservoir for Magnetic Separation and Quantum Dots-Based Immunolabeling of Metallothionein. Electrophoresis, 36, 1256-1264. https://doi.org/10.1002/elps.201400559 